\title{
Grupo de Medicina de Família e Comunidade de Santos: relato de experiência
}

\author{
Family and Community Medicine Group from Santos: Experience report \\ Grupo de Medicina Familiar y Comunitaria de Santos: Reporte de una experiencia
}

\author{
Paula Marcela Vilela Castro. Faculdade de Ciências Médicas de Santos (FCMS-UNILUS). pmarcela_13@hotmail.com (Autor correspondente) \\ Mayco José Reinaldi Serra. Faculdade de Ciências Médicas de Santos (FCMS-UNILUS). mayco_serra@hotmail.com \\ Talita Quadrado Penati. Faculdade de Ciências Médicas de Santos (FCMS-UNILUS). ta_penatti@yahoo.com.br
}

\section{Resumo}

Objetiva-se a discussão de diversos aspectos relacionados à Medicina de Família e Comunidade e das experiências vividas dentro da Associação Acadêmica Grupo de Medicina de Família e Comunidade de Santos (GMFC), do Centro Universitário Lusíada (UNILUS), vinculado à Faculdade de Ciências Médicas de Santos, São Paulo, Brasil. 0 GMFC busca a reflexão dos acadêmicos sobre o que é a Medicina de Família e Comunidade (MFC) enquanto especialidade, a fim de desmistificar ideias errôneas sobre a área em questão. Por meio de reuniões teóricas, congressos, jornadas e pesquisas científicas, o grupo busca consolidar uma visão concreta e íntegra de conceitos teóricos e aplicá-los na comunidade da Baixada Santista. A experiência do GMFC aprimora o conhecimento teórico-prático em MFC, favorecendo a qualificação dos acadêmicos para atuarem na prestação de serviços de saúde com aplicabilidade na Atenção Primária à Saúde (APS). Além disso, consolida-se 0 aprendizado acerca dos princípios do Sistema Único de Saúde (SUS) e da relevância de uma Medicina centrada na pessoa, com ênfase em uma visão holística da prática médica.Conclusão: A efetiva disseminação do conhecimento em MFC em nossa Universidade e o reconhecimento de que a MFC é importante para a formação generalista são grandes obstáculos constantemente enfrentados pelo grupo. Portanto, é de grande relevância a existência de um grupo acadêmico como 0 Grupo de Medicina de Família e Comunidade de Santos, pois além de integrar pessoas que se preocupam com uma abordagem diferenciada da Medicina, dissemina cada vez mais, dentro da universidade, o real significado da Medicina de Família e Comunidade.

\section{Abstract}

The purpose of this study is to discuss a wide spectrum of themes related to Family and Community Medicine (FCM) and the experiences within the "Associação Acadêmica Grupo de Medicina de Família e Comunidade de Santos" (GMFC) - a family and community medicine group of the "Centro Universitário Lusíada" (UNILUS), linked to the "Faculdade de Ciências Médicas de Santos", state of São Paulo, Brazil. The GMFC seeks academic reflection on the role of Family and Community Medicine as a medical specialty in order to demystify wrong ideas about the area in question. Through theoretical meetings, travels and scientific researches, we try to consolidate a concrete and thorough view of theoretical concepts and apply them to the community from the "Baixada Santista" region. The experience of the GMFC improves the theoretical and practical knowledge on FCM, promoting the qualification of academicians to operate in the provision of health services with applicability on Primary Heath Care. Moreover, the knowledge about the principles of the Brazilian Unified Health System (SUS) and the relevance of person-centered medicine are consolidated emphasizing the holistic view of medical practice. Conclusion: The effective dissemination of knowledge in FCM in our university and the recognition of its importance to the academic generalist education are obstacles constantly faced by the group. Therefore, the existence of such academic group is relevant because, in addition to integrating people who are concerned about a differentiated medicine approach, it increasingly spreads the real meaning of Family and Community Medicine in the university.

\section{Resumen}

Se objetiva discutir diversos aspectos de la Medicina Familiar y Comunitaria y de las experiencias vividas en el ámbito de la Asociación Académica Grupo de Medicina de Familia y Comunidad de Santos (GMFC), del Centro Universitario Lusíada (UNILUS), vinculado a la Facultad de Ciencias Médicas de Santos, São Paulo, Brasil. El GMFC busca la reflexión de los académicos sobre la naturaleza de la Medicina Familiar y Comunitaria (MFC) como especialidad, con el objetivo de desmitificar ideas erróneas sobre dicha área. Por medio de reuniones teóricas, congresos, jornadas e investigaciones científicas, el grupo busca consolidar una visión concreta e íntegra de conceptos teóricos y aplicarlos en la comunidad de la Bajada Santista. La experiencia del GMFC mejora el conocimiento teórico-practico en MFC promoviendo la capacitación de los académicos para actuar en la prestación de servicios de salud con aplicación en la Atención Primaria de la Salud (APS). Además, se consolida el aprendizaje acerca de los principios del Sistema Único de Salud (SUS) y de la relevancia de una medicina centrada en la persona, con énfasis en una visión holística de la práctica médica. Conclusión: La efectiva diseminación del conocimiento en MFC en nuestra universidad y la asimilación de que ésta es importante para la formación académica generalista son grandes obstáculos constantemente enfrentados por el grupo. Por lo tanto, resulta de gran relevancia la existencia de un grupo académico de este tipo, ya que, además de integrar personas que se interesan por un enfoque diferenciado de la medicina, disemina cada vez más, dentro de la universidad, el verdadero significado de la Medicina Familiar y Comunitaria.

Como citar: Castro PMV, Serra MJR, Penati TQ. Grupo de Medicina de Família e Comunidade de

Santos: relato de experiência. Rev Bras Med Fam Comunidade. 2013;8(26):16-9.

Disponível em: http://dx.doi.org/10.5712/rbmfc8(26)442
Palavras-chave: Medicina de Família e Comunidade Estudantes de Medicina Atenção Primária à Saúde Ligas Acadêmicas

Keywords:

Family and Community Medicine Medical, Students Primary Health Care Academic Leagues

Palabras clave: Medicina Familiar y Comunitaria Estudiantes de Medicina Atención Primaria de la Salud Ligas Académicas 


\section{Introdução}

Atualmente observa-se a manutençáo, por vezes, de condutas médicas que remetem ao modelo educacional hospitalocêntrico vigente desde o século XX, fortemente influenciado pelo flexnerismo ${ }^{1}$. Esse modelo recebe inúmeras críticas, pois considera que o aprendizado teórico-prático da academia deve centrar-se estritamente na doença. Nas palavras do próprio Flexner: "o estudo da Medicina deve ser centrado na doença de forma individual e concreta" ${ }^{2}$. Nesse modelo, aspectos sociais e coletivos, o público e a comunidade não contam para o ensino médico e não são considerados implicados no processo de saúde-doença ${ }^{3}$. No entanto, a integralidade na atençáo à saúde, juntamente com o desenvolvimento humanístico e a relaçáo médico-pessoa-comunidade, são de fundamental importância para a estruturação do conhecimento médicó .

A MFC sofre com conceitos errôneos acerca da formação médica necessária para exercer a especialidade, com seu surgimento recente, com o status social atribuído, a caracterização de exclusividade pública e comunitária da especialidade, a excessiva carga de trabalho dos profissionais que nela atuam e com a precariedade dos vínculos trabalhistas com eles estabelecidos, além da dificuldade de se trabalhar em equipes multiprofissionais ${ }^{4}$. Ademais, a especialidade é avaliada no meio acadêmico como realização de uma medicina desprovida de conhecimento, destinada apenas aos mais carentes, e que muitos estudantes vêem com desprezo ${ }^{5}$. Nesse sentido, o GMFC busca desmistificá-la e desistigmatizá-la, ao menos para os integrantes do grupo.

No Brasil, várias escolas médicas se propuseram a reformar seu ensino nos últimos 40 anos, tendo como perspectiva a construção de um novo paradigma, que busca responder à necessidade de mudanças nas relaçôes entre prática médica, organização da assistência à saúde e comunicação entre médicos e pacientes ${ }^{6}$.

Devido a esse cenário, os profissionais de saúde precisam se reorganizar e reavaliar conhecimentos, práticas e atitudes, visando desenvolver as competências necessárias à adequação das demandas (espontânea e programática) no sistema público de saúde. Isso porque o processo de ensino nas faculdades de Medicina do Brasil náo está voltado para a longitudinalidade do atendimento, mas apenas para a resolutibilidade dos processos de doença, o que exige dos acadêmicos uma formação médica diferente. Essa verticalização já não se adequa mais à realidade brasileira, pois após a criação do Programa Saúde da Família, em 1994, a atençâo integral à família e às comunidades passou a ter grande importância e melhorou muito a qualidade de vida dessas ${ }^{7,8}$.

Nesse sentido, a busca por uma Medicina mais humanizada e integral levou muitos estudantes a repensarem suas ambiçóes e objetivos na carreira médica. Assim, surgiram ideias para a fundação de grupos acadêmicos relacionados à Medicina de Família e Comunidade (MFC) cuja finalidade não é somente o atendimento continuado e humanizado das populaçóes, mas também um aprendizado teórico-prático e reflexôes em relação à saúde pública e à profissão.

Diversos discentes do país firmaram esses mesmos princípios e fundamentos para seus grupos, nas respectivas universidades, mas infelizmente a maioria não tem apoio institucional e, por isso, não consegue levar adiante esse propósito.

\section{Desenvolvimento}

A Associação Acadêmica Grupo de Medicina de Família e Comunidade de Santos (GMFC), uma associação de acadêmicos de Medicina vinculada à Faculdade de Ciências Médicas de Santos (FCMS), do Centro Universitário Lusíada (UNILUS), foi fundada em maio de 2008. Ela surgiu a partir de iniciativas de estudantes preocupados com a necessidade da existência, na Região Metropolitana da Baixada Santista, de um órgão destinado à difusão de conhecimentos na área de MFC que também contribuisse para a complementação da formação acadêmica. Trata-se de um grupo aberto aos estudantes da área de saúde da mesma Universidade, entretanto há um número fixo de acadêmicos de Medicina que se renovam anualmente, de acordo com a demanda e o interesse.

A organização administrativa do GMFC é dividida em Assembléia geral, Diretoria, Conselho fiscal e Departamentos. A Assembléia geral é órgão máximo, constituído por todos os integrantes, com finalidade de eleger anualmente a Diretoria e resolver pendências administrativas, quando necessário. A Diretoria, por sua vez, é constituída por presidente, vice-presidente, $1^{\circ}$ secretário, $2^{\circ}$ secretário e tesoureiro. O Conselho fiscal, indicado pela Assembléia geral, é composto por três membros, que náo podem integrar a Diretoria, e tem por objetivo fiscalizar o cumprimento do estatuto. Ademais, sete Departamentos atuam de forma autônoma em prol do aprimoramento do GMFC, são eles: Departamento de aprendizado teórico, Departamento científico, Departamento de inserção na comunidade, Departamento de valores humanos, Departamento de integração, Departamento de relaçóes externas e Departamento de publicidade e tecnologias. 
Nos últimos quatro anos, o GMFC realiza reunióes semanais em que ocorrem palestras sobre temas pertinentes de MFC, elaboradas e apresentadas pelos próprios acadêmicos integrantes do grupo, professores ou médicos de família e comunidade convidados, seguidas por uma atividade dinâmica na qual o tema é discutido, a qual tem se demonstrado sempre uma experiência produtiva e agregadora ao conhecimento acadêmico. Esporadicamente, as palestras semanais são substituídas por documentários sobre a saúde pública do país, o Sistema Único de Saúde (SUS) e a MFC.

Pelo menos uma vez por semestre, atividades nas quais tem sido possível, de forma relevante, colocar em prática conhecimentos acadêmicos da especialidade e desenvolver maior sensibilidade na relação médico-pessoa-comunidade são realizadas na comunidade.

A jornada científica do grupo é realizada anualmente, envolvendo acadêmicos de Medicina e professores da faculdade, que assistem a palestras que exploram os conceitos de MFC e disseminam a política da Estratégia Saúde da Família (ESF), sendo esse evento importante para a propagaçáo desse conhecimento no meio acadêmico.

Outro ponto a se destacar é a participação dos integrantes do grupo em congressos, a qual, de forma gratificante, tem sido excepcional. Além de todo conhecimento obtido, os participantes têm inscrito pôsteres de cunho informativo e reflexivo sobre o grupo.

Desde a criação do GMFC foi possível observar uma queda significativa no número de alunos que o integravam, sendo talvez a curiosidade o motivo maior para a adesão ao grupo na época de sua fundação. Porém, com o passar do tempo, o desinteresse surgiu, muito pela estruturaçáo inadequada da disciplina na Universidade e pelo preconceito acerca do que realmente seria a MFC. Nos últimos dois anos, procurou-se quebrar esse paradigma intensificando-se as açóes em prol do grupo, ou seja, buscando maior representatividade dentro da própria Universidade e despertando interesse dos alunos, o que vem sendo conquistado de forma satisfatória.

Percebe-se situação semelhante na questão da participação nas jornadas científicas organizadas pelo grupo. Entretanto, dentre aqueles alunos que já integravam o GMFC e que participaram de congressos de MFC desenvolveu-se uma maior motivação pessoal e acadêmica, o que amplia a perspectiva dessas pessoas e, consequentemente, do grupo. Isso porque esses acreditam que a MFC é mais humanizada, longitudinal e focada na atenção primária.

Talvez esse outro ponto ajude a explicar o desinteresse dos acadêmicos pelo grupo, já que atualmente a Medicina em geral se encontra fragmentada e subespecializada, favorecendo práticas curativistas e reabilitadoras que comprovadamente são mais rentáveis9. Além disso, existem ainda inúmeras dificuldades financeiras e administrativas que tornam-se barreiras para a prática da MFC no Brasil - desde a ausência de equipes multiprofissionais engajadas na atenção ao paciente até salários incompatíveis e condiçóes de trabalho inadequadas ao escopo da especialidade.

Carole et al. ${ }^{10}$ levantaram determinantes da escolha pela Atenção Primária à Saúde (APS) entre estudantes de Medicina, tendo encontrado como correlação direta com essa escolha: estágios obrigatórios em Medicina de Família e Comunidade e experiências longitudinais (com mais de um ano de duração) em APS. Entre os fatores que desestimulam a procura pela APS destacam-se a carência de conhecimento sobre o assunto e as imagens distorcidas da especialização nessa área, adquiridas ao longo da graduação e fortemente influenciadas pela cultura da instituição ${ }^{5}$. Desse modo, as reunióes semanais do grupo acadêmico comprovam a importância de discussóes nesse sentido e sua inserção gradativa no dia a dia dos alunos.

Observa-se que muitos integrantes do grupo ainda não manifestaram interesse em, futuramente, especializar-se em Medicina de Família e Comunidade. Entretanto, o fato de estarem sendo abordados, continuamente, os preceitos fundamentais de uma Medicina centrada na pessoa e não na doença poderá talvez refletir-se em uma prática futura que promova impactos positivos na saúde dos pacientes, além do fortalecimento do vínculo médico-paciente, independentemente da especialidade que o acadêmico desejar seguir.

\section{Conclusão}

A partir das experiências vividas no Grupo de Medicina de Família e Comunidade do Centro Universitário Lusíada torna-se possível uma reflexão acerca do cuidado das pessoas, com o objetivo de se desenvolver uma Medicina sensibilizada a fornecer ao paciente um cuidado integral. Isso se traduz não apenas na Medicina destinada a cessar patologias, mas capaz de ir além da queixa referida como promotora de desconforto e carência de saúde, a fim de contemplar o ser humano como um todo. 
De maneira similar, as palestras e dinâmicas de integração desenvolvidas entre os membros do grupo durante as reuniōes, assim como as atividades práticas de intervenção nas comunidades, estimulam as relaçóes interpessoais entre os acadêmicos. Essas atividades favorecem uma abordagem do paciente capaz de estimular os estudantes a lidarem melhor com possíveis sentimentos de timidez e insegurança ao abordar aquele que espera por auxílio e palavras de conforto.

Portanto, é de grande importância a disseminação de grupos acadêmicos como esse, pois além de integrar pessoas que se preocupam com uma abordagem diferenciada da Medicina, promove cada vez mais, dentro da Universidade, o real significado da Medicina de Família e Comunidade.

\section{Referências}

1. Restrepo JFP. Abraham Flexner y el flexnerismo: fundamento imperecedero de la educación médica moderna. Medicina (Bogotá). 1998; 20(48): 6-14.

2. Flexner A. Medical Education in the United States and Canada. Carnegie Foundation for The Advancement of Teaching; 1910 . Bulletin 4.

3. Santos JO. Filosofia da Educação Médica: interpretação da práxis. Rev Bras Educ Med. 1986; 10(2): 82-6.

4. Mello GA, Mattos ATR, Souto BGA, Fontanella BJB, Demarzo MMP. Médico de família: ser ou não ser? Dilemas envolvidos na escolha desta carreira. Rev Bras Educ Med. 2009; 33(3): 464-471. http://dx.doi.org/10.1590/S0100-55022009000300017

5. Gonçalves RJ, Soares RA, Troll T, Cyrino EG. Ser médico no PSF: formação acadêmica, perspectivas e trabalho cotidiano. Rev Bras Educ Med. 2009; 33(3): 393-403. http://dx.doi.org/10.1590/S0100-55022009000300009

6. Cabral Filho WR, Ribeiro VMB. A escolha precoce da especialidade pelo estudante de medicina: um desafio para a educação médica. Rev Bras Educ Med. 2004; 28(2): 133-144.

7. McWhinney IR, Freeman T. Manual de Medicina de família e comunidade. 3. ed. São Paulo: Artmed editora. 2010.

8. Stewart M. Medicina centrada na pessoa: Transformando o método clínico. 2. ed. São Paulo: Artmed editora. 2010.

9. Bonfada D, Cavalcante JRLP, Araujo DP, Guimaraes J. A integralidade da atenção à saúde como eixo da organização tecnológica nos serviços. Ciênc Saúde Coletiva. 2012; 17(2): 555-560. http://dx.doi.org/10.1590/S1413-81232012000200028

10. Carole JB, Meurer LN, Maldonado G. Determinants of primary care specialty choice: a non-estatistical meta-analysis of the literature. Acad Med. 1995; 70(7): 620-41. http://dx.doi.org/10.1097/00001888-199507000-00013 